\title{
Gold Nanostar-Coated Polystyrene Beads as Multifunctional Nanoprobes for SERS Bioimaging
}

Ana B. Serrano-Montes, ${ }^{\dagger}$ Judith Langer, ${ }^{\dagger}$ Malou Henriksen-Lacey, ${ }^{\dagger, \mathbb{I}}$ Dorleta Jimenez de Aberasturi, ${ }^{\dagger}$, I

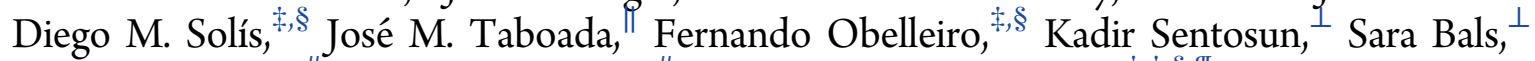
Ahmet Bekdemir, ${ }^{\#}$ Francesco Stellacci, ${ }^{\#}$ and Luis M. Liz-Marzán ${ }^{*}, \dagger, \ddagger, \S, \mathbb{I}$

${ }^{\dagger}$ CIC biomaGUNE, Paseo de Miramón 182, 20009, Donostia - San Sebastian, Spain

${ }^{\ddagger}$ Ikerbasque, Basque Foundation for Science, 48013 Bilbao, Spain

${ }^{\S}$ Dept. Teoría de la Señal y Comunicaciones, University of Vigo, 36301 Vigo, Spain

"Dept. Tec. Computadoras y Comunicaciones, University of Extremadura, 10003 Cáceres, Spain

${ }^{\perp}$ EMAT-University of Antwerp, Groenenborgerlaan 171, B-2020 Antwerp, Belgium

"\# Institute of Materials, Ecole Polytechnique Fédérale de Lausanne, 1015 Lausanne, Switzerland

${ }^{\mathbb{I}}$ Biomedical Research Networking Center in Bioengineering, Biomaterials, and Nanomedicine (CIBER-BBN), 20009 Donostia - San Sebastian, Spain

\section{Supporting Information}

ABSTRACT: Hybrid colloidal nanocomposites comprising polystyrene beads and plasmonic gold nanostars are reported as multifunctional optical nanoprobes. Such self-assembled structures are excellent Raman enhancers for bioapplications as they feature plasmon modes in the near-infrared "first biological transparency window”. In this proof of concept study, we used 4mercaptobenzoic acid as a Raman-active molecule to optimize the density of gold nanostars on polystyrene beads, improving SERS performance and thereby allowing in vitro cell culture imaging. Interestingly, intermediate gold nanostar loadings were found to yield higher SERS response, which was confirmed by electromagnetic modeling. These engineered hybrid nanostructures notably improve the possibilities of using gold nanostars as SERS tags. Additionally, when fluorescently labeled polystyrene beads are used as colloidal carriers, the composite particles can be applied as promising tools for multimodal bioimaging.

\section{INTRODUCTION}

Surface-enhanced Raman scattering (SERS) is a powerful spectroscopic technique that has attracted large interest due to numerous advantages over conventional analytical tools. ${ }^{1}$ It provides nondestructive characterization, high sensitivity even down to single molecule detection, ${ }^{2,3}$ and multiplexed capability based on narrow and sharp molecule-specific vibrational Raman peaks. ${ }^{4}$ SERS relies on the unique optical properties of metallic nanostructures, which can support high electromagnetic fields upon excitation of localized surface plasmon resonances (LSPRs), in turn enhancing the Raman scattering of adsorbed molecules. Recent work has focused on the development of novel plasmonic nanostructures for SERS (usually known as SERS substrates). In particular, gold nanoparticles ( $\mathrm{Au} \mathrm{NP}$ ) are extensively used due to versatile synthesis methods, long-term stability, and biocompatibility. ${ }^{5}$ The ideal SERS substrate should be reproducible and chemically stable and exhibit high and spatially uniform SERS efficiency. Extremely high electromagnetic (EM) field enhance-

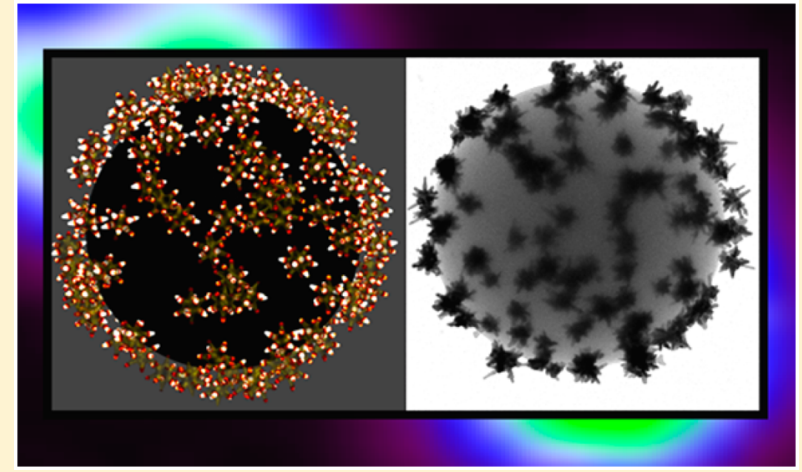

ments (also named "hot spots") are produced at the junctions between adjacent metal nanoparticles, ${ }^{6}$ as well as at sharp edges in anisotropic nanostructures. ${ }^{7-9}$ Among the latter, gold nanostars (Au NSs) are of especial interest since their optical responses can be readily tuned within the visible and nearinfrared (NIR) regions, and their spikes act as efficient nanoantennas so that high EM fields are generated at the tips, giving rise to multiple intrinsic hot spots within a single nanoparticle. ${ }^{10,11}$ Various Au NS-based SERS substrates have been reported for chemical detection. Extremely high Raman signals have been obtained for dithiolated Raman-active molecules trapped between Au tips and a smooth gold film, ${ }^{9}$ which were explained due to enhanced confinement effects. ${ }^{12}$

Special Issue: Richard P. Van Duyne Festschrift

Received: March 3, 2016

Revised: May 18, 2016

Published: May 20, 2016 
An interesting alternative for practical applications comprised the immobilization of $\mathrm{Au}$ NSs on either gold or flexible polydimethylsiloxane substrates for chemical sensing of analytes that can directly interact with the Au NS surface. ${ }^{3,14}$ Alternatively, colloidal templates have been also widely employed as plasmonic supports. Polystyrene (PS)-based plasmonic SERS substrates can be easily manipulated, are resistant to particle aggregation, and may display high SERS activity. ${ }^{15}$ PS is one of the most commonly used polymeric materials due to its low toxicity, high water stability, and easy synthesis and surface functionalization. ${ }^{16}$ Although in situ growth of nanoparticles onto the surface of PS microspheres has been widely reported, ${ }^{17-20}$ significantly better control over the desired NP size and shape can be achieved when Au NPs are presynthesized and subsequently assembled onto the polymer surface. $^{21-23}$

Herein we report the assembly of Au NSs onto PS beads with tunable Au NS density, which can be used to optimize the SERS efficiency of these hybrid nanomaterials. We additionally employed highly detailed electromagnetic modeling as a complementary tool to analyze and select the most efficient configuration of these hybrid nanostructures toward applications in cell bioimaging. In this context, we propose the combination with fluorescence imaging. The integration of two functionalities in the same composite particle is proposed to compensate the main deficiencies of both techniques and constitutes a major strategy to independently track and image objects using different optical tools. ${ }^{24}$ Although fluorescence is a well-established technique that allows fast imaging acquisition, ${ }^{25}$ fluorophores can show spectral overlap, photobleaching and are typically limited to visible light excitation. ${ }^{26}$ On the other hand, SERS offers the possibility of extended multiplexing and using excitation wavelengths at the so-called "biological transparency window". ${ }^{27}$ In this respect, fluorescent-SERS tags have been successfully used for imaging of duplex coexpressed markers on cancer cells, ${ }^{28}$ as well as for simultaneous multimodal tumor detection and photodynamic therapy in vivo. ${ }^{29}$ Although most of the reported strategies comprise the conjugation of a fluorophore onto the plasmonic core, ${ }^{30-32}$ we alternatively propose the use of fluorescently labeled PS beads as carriers to attach the SERS-active Au NSs. Compared to previously reported fluorescent-SERS dual mode tags, our multifunctional nanoprobes are more robust due to the use of PS supports, ${ }^{15}$ and the colocalization of the plasmonic nanostars on the colloidal structure allows the detection of the intracellular SERS signal. Additionally, NIR excitation can be used and silica coating is avoided, so that the stability in biological media can be notably improved. ${ }^{33,34}$ We demonstrate that our designed nanoassemblies are promising nanotools for multimodal bioimaging combining three different spectroscopic methods, namely, fluorescence, light scattering, and SERS.

\section{MATERIALS AND METHODS}

Materials. Milli-Q water (resistivity $18.2 \mathrm{M} \Omega \cdot \mathrm{cm}$ ) was used in all experiments. Hydrogen tetrachloroaurate trihydrate $\left(\mathrm{HAuCl}_{4} \cdot 3 \mathrm{H}_{2} \mathrm{O}, \geq 99.9 \%\right)$, sodium citrate tribasic dihydrate $(\geq 98 \%)$, silver nitrate $\left(\mathrm{AgNO}_{3}, \geq 99 \%\right)$, L-ascorbic acid (AA, $\geq 99 \%$ ), O-[2-(3-mercaptopropionylamino)ethyl]- $O^{\prime}$-methylpolyethylene glycol (PEG-SH, Mw $5000 \mathrm{~g} / \mathrm{mol}$ ), and 4mercaptobenzoic acid (4-MBA, 90\%), were purchased from Sigma-Aldrich. Hydrochloric acid solution $(\mathrm{HCl}, 37 \%)$ was purchased from Panreac. Fluorescently labeled amino-functionalized PS beads $\left(\lambda_{\mathrm{ex}} / \lambda_{\mathrm{em}}=477 \mathrm{~nm} / 519 \mathrm{~nm}\right.$; NP size $483 \pm 3$ nm) were purchased from IKERLAT Polymers (Lasarte, Spain). Nonlabeled carboxylic acid-functionalized PS beads were a gift from the same company. All glassware was washed with aqua regia, rinsed 3-fold with milli-Q water, and dried before use. Dulbecco's modified Eagle's medium (DMEM), fetal bovine serum (FBS), penicillin-streptomycin (PStrep), trypsin-EDTA, wheat germ agglutinin-AF647 (WGA647), and 4,6-diamidine-2-phenylindole dihydrochloride (DAPI) were purchased from Invitrogen. MTT cell proliferation kit was purchased from Roche.

Sample Characterization. Transmission electron microscopy (TEM) images were collected with a JEOL JEM1400PLUS TEM operating at $120 \mathrm{kV}$, using carbon coated 400 square mesh copper grids. HAADF-STEM images and electron tomography tilt series were acquired using a FEI Osiris electron microscope operated at $200 \mathrm{kV}$. For the reconstruction of the series the SIRT algorithm was used, as implemented in the ASTRA toolbox. ${ }^{35} \mathrm{UV}-$ Vis-NIR optical extinction spectra were recorded using an Agilent $8453 \mathrm{UV}-$ vis diode-array spectrophotometer.

Synthesis of Gold Nanostars. Au NSs were prepared by a modified seed-mediated growth method. ${ }^{36}$ Briefly, the seed solution was prepared by adding $5 \mathrm{~mL}$ of a $1 \%$ citrate solution to $95 \mathrm{~mL}$ of boiling $0.5 \mathrm{mM} \mathrm{HAuCl} \mathrm{H}_{4}$ solution under vigorous stirring. After $15 \mathrm{~min}$ of boiling, the solution was cooled down to room temperature and then kept at $4{ }^{\circ} \mathrm{C}$ for long-term storage. The as-synthesized Au nanoparticle seeds had an LSPR maximum at $519 \mathrm{~nm}$. For Au NS synthesis, $500 \mu \mathrm{L}$ of the citrate-stabilized seed solution was added to $10 \mathrm{~mL}$ of $\mathrm{HAuCl}_{4}$ $(0.25 \mathrm{mM})$ solution containing $10 \mu \mathrm{L}$ of $\mathrm{HCl}(1.0 \mathrm{M})$ in a 20 $\mathrm{mL}$ glass vial at room temperature, under moderate stirring. Quickly, $100 \mu \mathrm{L}$ of $\mathrm{AgNO}_{3}(3 \mathrm{mM})$ and $50 \mu \mathrm{L}$ of AA (100 $\mathrm{mM}$ ) were added simultaneously to the above solution. The solution rapidly turned from light red to greenish indicating the formation of Au NSs. Immediately after synthesis, the solution was stirred with PEG-SH (final concentration $8 \times 10^{-7} \mathrm{M}$ ) for $15 \mathrm{~min}$, washed by centrifugation $\left(1190 \mathrm{~g}, 25 \mathrm{~min}, 10^{\circ} \mathrm{C}\right)$, and redispersed in water. The $\mathrm{Au}^{0}$ concentration of the $\mathrm{Au}$ NS solution was adjusted to $0.5 \mathrm{mM}$.

Assembly of Au NSs and PS Beads. A simple procedure was used to form Au NS-coated PS particles. $1 \mathrm{~mL}$ of PEGstabilized Au NS solution $0.5 \mathrm{mM}$ was added dropwise onto $100 \mu \mathrm{L}$ of PS in an Eppendorf tube under sonication, and the particle mixture was incubated overnight. Three different initial PS concentrations $(0.15,0.3$, and $1 \%$ in solids $)$ were used to obtain high, medium, and low Au NS loading, respectively. The unbound $\mathrm{Au}$ NSs were removed by two centrifugation steps $\left(1770 \mathrm{~g}, 3 \mathrm{~min}, 20{ }^{\circ} \mathrm{C}\right)$, and the samples were redispersed in Milli-Q water adjusting the concentrations to $1.5 \times 10^{-2}$ (PS, \% in solids).

Cell Culture. Human lung epithelial cancer (A549), breast cancer (MCF-7), and murine monocyte-macrophage (J774) cell lines were grown in DMEM supplemented with 10\% FBS and $1 \%$ PStrep (known as cDMEM). Cells were passaged using Trypsin-EDTA. For SERS, confocal and dark field microscopy between $5 \times 10^{4}$ to $2 \times 10^{5}$ cells were plated in glass bottomed dishes with marked grids ("Grid-50", Ibidi) and allowed to adhere overnight. Fluorescently labeled Au NS-PS particles were added to cells at a final concentration of PS from $7.5 \times$ $10^{-4}$ to $3.75 \times 10^{-4} \%$ in solids, depending on the experiment setup, and left for various time points at $37^{\circ} \mathrm{C}$ to allow particle uptake. 
Confocal Fluorescence and Dark-Field Microscopy.

Particle uptake in A549 cells was imaged using a Zeiss LSM confocal microscope with $477 \mathrm{~nm}$ excitation and a 500-550 $\mathrm{nm}$ BP filter. Either $40 \times(\mathrm{NA}=1.3)$ or $63 \times(\mathrm{NA}=1.4)$ objectives were used. Multichannel (transmission and fluorescence) Z-stacks were taken using ZEN software. For dark field microscopy (DFM) images, cells were fixed using $4 \%$ formaldehyde (20 min, room temperature) and mounted using a \#1.5 coverslip and mounting fluid (Dako). Once dry, the dish was inverted and viewed using a Nikon Eclipse $\mathrm{Ti}-\mathrm{U}$ microscope equipped with a $60 \times(\mathrm{NA}=0.7)$ objective and oil condenser. Neutral density and neutral color balance filters were inserted. Photos were taken using a $0.1 \mathrm{~s}$ exposure and light intensity levels, and other variables were unchanged for the different samples.

Cell Viability. Nanoparticle induced cytotoxicity was measured using the MTT assay. A549 cells were plated in 96 well plates at $1 \times 10^{4}$ cells/well and allowed to adhere before adding NPs at final PS concentrations ranging from $1.5 \times 10^{-3}$ to $9.4 \times 10^{-5} \%$ in solids. Cells were incubated for $66 \mathrm{~h}$ before removal of the cell medium and addition of MTT reagent (1/ 20 dilution in cDMEM). Cells were incubated for $1.5 \mathrm{~h}$ at $37{ }^{\circ} \mathrm{C}$, followed by removal of the supernatant and addition of DMSO to lyse cells. Absorbance was read at $550 \mathrm{~nm}$, and cell viability calculated as a \% of the control.

SERS Measurements. SERS was measured with a confocal Raman microscope (Renishaw inVia) equipped with $1024 \times$ 512 CCD detector using a $785 \mathrm{~nm}$ laser excitation source (maximum output of $250 \mathrm{~mW}$ ) and a $1200 \mathrm{l} / \mathrm{mm}$ diffraction grating. For reference and test SERS experiments, $1 \mathrm{~mL}$ of 4MBA labeled Au NS-PS beads (low, medium, and high Au NS loading, final 4-MBA concentration $1 \mathrm{mM}$ ) was placed in a $1 \mathrm{~mL}$ glass vial (Thermo Fisher), and spectra were collected using a $10 \times$ objective $(\mathrm{NA}=0.35)$ in expanded scan mode with an integration time of $10 \mathrm{~s}$ and a laser power of $18 \mathrm{~mW}$. SERS measurements in cells were carried out in static mode (center of scattered wavenumber $1200 \mathrm{~cm}^{-1}$ ) with an integration time of $1 \mathrm{~s}$ and a laser power of $15 \mathrm{~mW}$ using a water immersion $40 \times$ objective $(\mathrm{NA}=0.85)$. Maps were generated by plotting the intensity of the 4-MBA ring deformation $\left(1078 \mathrm{~cm}^{-1}\right)$ as a function of grid position. For estimation of the SERS enhancement factor, 4-MBA labeled Au NS-PS beads (low, medium, and high, $50 \mu \mathrm{L}$ ) were dropped onto glass slides, and a thin film was formed by spin coating (30 s, $33 \mathrm{rps}$ ). Solid 4MBA was pestled and compacted onto a glass slide to form a homogeneous, thick layer. SERS and Raman spectra were measured with integration time of $10 \mathrm{~s}$ (extended mode) at laser power of $1.7 \mathrm{~mW}$ using a $50 \times$ objective $(\mathrm{NA}=0.75)$. The average laser powers were measured in air after passing the laser beam through the objectives used in the corresponding experiments by means of a photodiode sensor (PD300-3W) and a power meter (Nova, both from Ophir).

Electromagnetic-Field and SERS Intensity Calculations. Spectral and near-field enhancement simulations were carried out using a numerical method based on a surface integral equation-method of moments (SIE-MoM) formulation $\left(\mathrm{M}^{3}\right.$ solver $){ }^{37,38}$ With this methodology, the metallic nanostructures are replaced by equivalent electric and magnetic currents placed over the particle boundary surfaces and interfaces, thus reducing the computation domain of any radiation or scattering problem to the surface boundaries of materials. Additionally, the SIE-MoM approach yields high stability, particularly when dealing with resonant metallic response, as the singular behavior of fields is analytically described by the Green's function and its derivatives. Through Maxwell's equations and the boundary conditions for the total fields, we derive a set of SIEs for the unknown equivalent currents. These SIEs are subsequently discretized by applying a Galerkin MoM procedure in terms of a set of basis and testing functions, leading to a dense $N \times N$ matrix system of linear equations (being $N$ the number of basis functions used to expand the unknown equivalent currents on the surface boundaries).

For a realistic simulation of the large-scale plasmonic systems considered here, SIE-MoM was accelerated via the multilevel fast multipole algorithm-fast Fourier transform (MLFMAFFT) ${ }^{39,40}$ providing a high algorithmic efficiency-computational cost of $O(N \log N)$-along with high-scalability via parallelization using multicore computer clusters.

The simulations were carried out on a workstation with four 15-core Intel Xeon E7-4880 V2 37.5 MB L3 cache processors at $2.5 \mathrm{GHz}$. A high surface mesh density was considered using up to 975840 basis functions for the most computationally intensive case (Au NS-PS particles with high loading), with an edge size ranging from $1 / 162$ wavelengths for the shortest simulated wavelength to $1 / 405$ for the longest. A relative error norm of $10^{-5}$ was considered to halt the Krylov iterative solver. Considering the above, the computation times ranged from 21 min to $1.5 \mathrm{~h}$ per wavelength, while the memory use ranged from 11 to $28 \mathrm{~GB}$.

\section{RESULTS AND DISCUSSION}

Monodispersed PS beads coated with Au NSs were prepared using a two-step procedure: PEG-coated Au NSs were first synthesized through a well-known seed-mediated growth method $^{36}$ and subsequently allowed to self-assemble onto the amine-terminated PS surface ${ }^{14}$ (Figure 1). This strategy leads to highly stable nanostructures and allows tight control over the final plasmonic performance. An alternative method, based on the adsorption of Au nanospheres on PS and in situ growth of gold tips, resulted in aggregation and growth of nonuniform particles containing branched structures of different sizes and shapes that protrude out from the polymer surface (Figure S1b, SI). It should also be noted that, when PS spheres functionalized with carboxylic acid were used, Au NSs did not bind to the polymer surface (Figure S1c, SI). ${ }^{22}$

Due to the strong affinity of amino groups with the gold surface, the polymer beads were densely covered with Au NSs, as confirmed by TEM (Figure 1a). In addition, annular dark field-scanning TEM (ADF-STEM) analysis was carried out for detailed analysis of individual self-assembled particles. As can be observed in Figure 2a a central polymer particle is surrounded by smaller and brighter objects that correspond to Au NSs. Electron tomography and three-dimensional (3D) reconstruction of the same particle provided additional information regarding $\mathrm{Au} \mathrm{NS}$ distribution. The visualization of 3D reconstruction (Figure $2 \mathrm{~b}$ ) shows that $\mathrm{Au}$ NSs (golden) are indeed adsorbed around the whole PS surface (blue), but the distribution is not completely uniform and a certain degree of aggregation is consistently observed. The full reconstruction movie is provided as Supporting Information.

The same method could be applied to other metal nanoparticles, such as PVP-stabilized $\mathrm{Au} \mathrm{NSs}{ }^{10}$ or silver nanoparticles (Ag NPs), ${ }^{41}$ as exemplified in Figure S2 (SI). It is important to note that the density of Au NSs per PS bead could be controlled by simply varying the concentration of PS 
i)
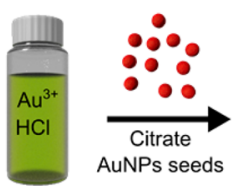

ii)

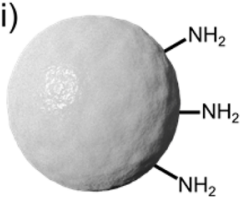

Fluorescent PS beads

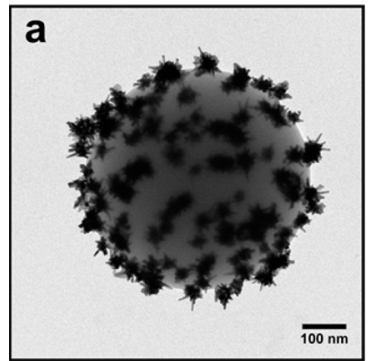

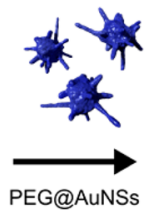

$\mathrm{AgNO}_{3}$ Ascorbic acid PEG-SH PEG-AU NSS
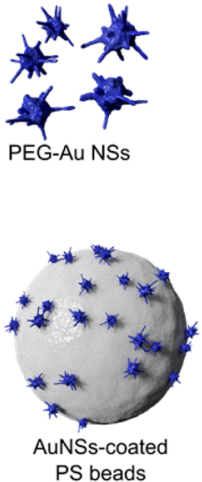

PS beads

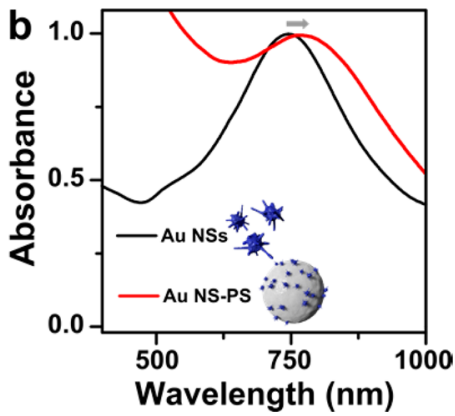

Figure 1. Upper panel: Schematic illustration of gold nanostar synthesis (i) and assembly (ii). Lower panel: representative TEM image of a Au NS-PS bead (a) and UV-vis-NIR spectra (b) of Au NS (black line) and Au NS-PS (red line) colloids.
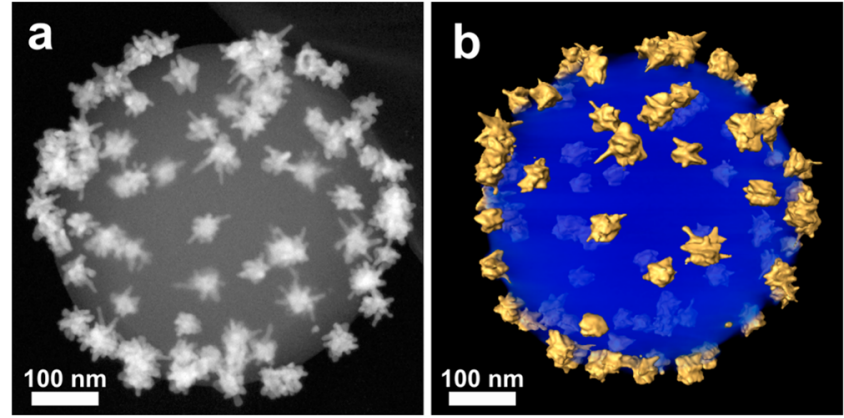

Figure 2. ADF-STEM (a) and 3D reconstruction (b) images of an $\mathrm{Au}$ NS-PS bead.

while keeping constant the Au NS concentration. Thus, PS spheres could be coated with low (Figure 3a), medium (Figure $3 \mathrm{~b}$ ), and high $\mathrm{Au}$ NS loadings (Figure 3c). It should be noted however that, when increasing excessively the Au NS concentration during the assembly process, Au NS aggregation was ultimately induced, and therefore uniform coatings could not be obtained (Figure S3, SI). The number of Au NS on the PS surface could be estimated by analyzing at least 20 TEM images, obtaining for low, medium, and high coatings an average of ca. 20, 80, and $120 \mathrm{Au}$ NSs per PS bead, respectively (Figure S4, SI). Again, as the density of Au NSs increased, so did the tendency to form small clusters on the PS core. The spiky morphology of the Au NSs gives rise to an LSPR band corresponding to tip plasmon modes, as well as a less intense shoulder from the core mode. ${ }^{42}$ A similar optical response was found for the Au NS-PS composite particles, with some differences (LSPR red-shift and broadening) which are due to the presence of the PS core (enhanced scattering at low

wavelengths and increased refractive index), but also from the close proximity between some Au NSs on the PS surface which leads to plasmon coupling effects (Figures $1 \mathrm{~b}$ and S2). ${ }^{12} \mathrm{We}$ also found that, when the Au NS density was increased, the intensity of the LSPR band also increased, as shown in Figure 3d. A similar trend was observed when calculating the extinction spectra of the assembled particles (Figure 3e). Trying to represent the Au NS-PS system in its full complexity, some degree of polydispersity was considered for the assembled $\mathrm{Au}$ NSs in the numerical simulations, which were modeled as 12 sharp tips branching out from a central spherical core. Based on representative TEM images of the Au NSs, a (normally distributed) branch length of $22 \pm 8 \mathrm{~nm}$ was considered, with a width of $15 \mathrm{~nm}$ at the base and a tip apex diameter of $4 \mathrm{~nm}$. The core diameter was fixed at $35 \mathrm{~nm}$. Regarding the PS sphere, the diameter was set to $483 \mathrm{~nm}$. The Au NSs were randomly distributed around the PS beads, but some aggregation (slightly higher as the loading increases, see Figure 5) was induced, again based on TEM images. A minimum distance of $1 \mathrm{~nm}$, both between neighboring Au NSs and between $\mathrm{Au}$ NSs and the PS bead, was considered. With the above prescriptions, ten different models were simulated and averaged to obtain the spectrum in each of the three different density cases. The calculated spectra are in close agreement with the experimental results, with LSPR red-shift and broadening, as well as enhanced scattering at low wavelengths, due to the presence of the PS core and plasmon coupling effects between Au NSs.

One the other hand, large EM field confinements are expected within nanometer-sized gaps between neighboring $\mathrm{Au}$ NSs, which individually support hot spots at their tips. ${ }^{9,43}$ Therefore, high SERS signals are expected for Raman-active molecules trapped at such strong localized EM fields. ${ }^{44}$ We used 4-MBA as Raman reporter molecule, which provides high SERS signal under $785 \mathrm{~nm}$ excitation and is known to strongly bind to the Au surface. In this case, the presence of 4-MBA is particularly useful, as it has been not only been reported to prevent Au NS reshaping through thiol binding, ${ }^{45,46}$ but additionally the negative charges at the carboxylic groups oppose particle aggregation. ${ }^{47}$ The SERS response from 4MBA-labeled nanocomposites was measured and compared for particles with different Au NS loadings. Figure 4a depicts representative SERS spectra of 4-MBA from PS beads coated with low, medium, and high Au NS densities. As expected, the most prominent peaks appear at 1580 and $1078 \mathrm{~cm}^{-1}$ corresponding to $\nu(\mathrm{C}-\mathrm{C})$ ring stretch and ring breathing modes of the molecule, respectively. ${ }^{48-50}$ Remarkably, the sample with the highest Au NS density (high Au NS) did not result in the most intense SERS signal. A similar behavior has been previously reported for aggregated Au NSs and was explained by some sort of hot-spot deactivation due to interaction between tips with different orientations and geometries. ${ }^{47,51}$ On the one hand, this situation is in stark contrast to the aggregation-induced hot-spot formation between e.g. spherical nanoparticles or nanorods. ${ }^{52} \mathrm{~A}$ recent publication by our group also showed that a monolayer of densely packed spherical Au NPs generates a significantly higher SERS signal than a dense monolayer of Au NSs with similar particle size. ${ }^{46}$

The experimental enhancement factor (EF) for assemblies of different Au NS coverage can be calculated using the following equation: 

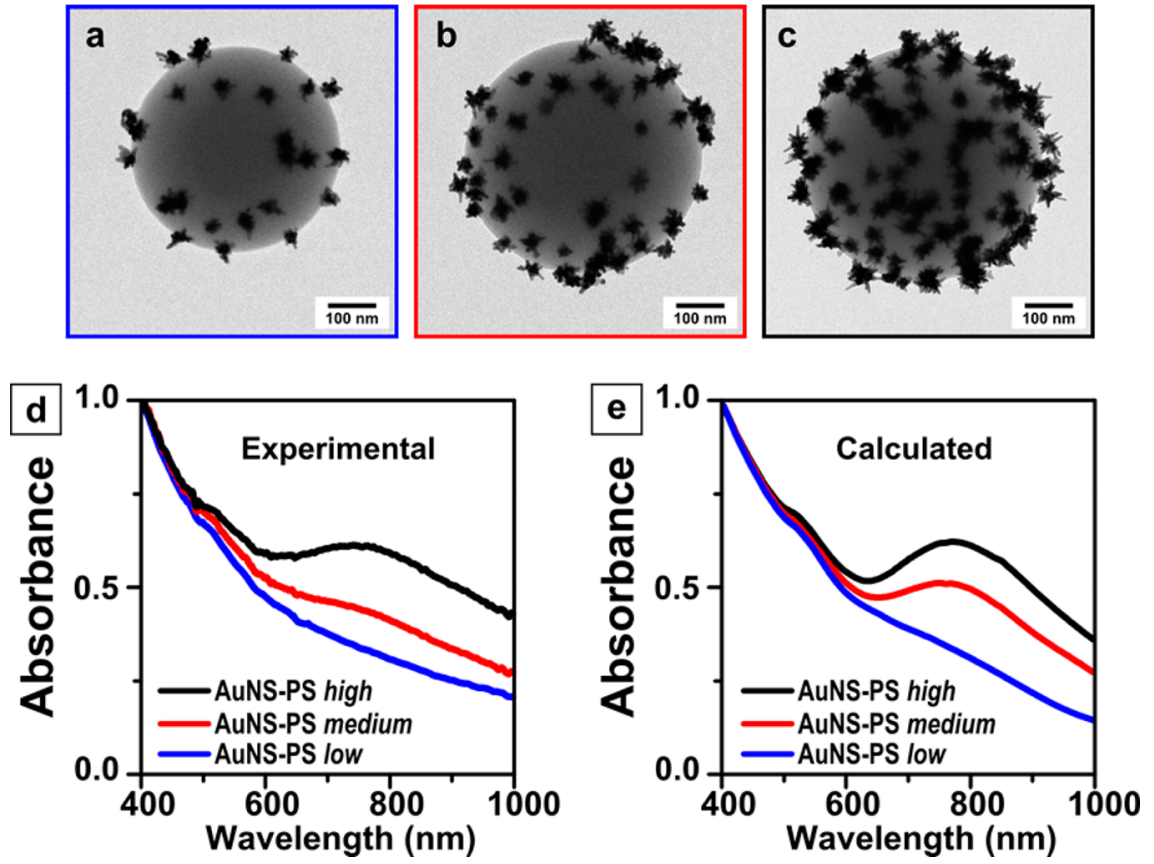

Figure 3. Representative TEM images $(a-c)$ and experimental (d) and calculated (e) Vis-NIR spectra of PS beads coated with different Au NS densities $($ blue $=$ low, red $=$ medium, and black $=$ high $)$.
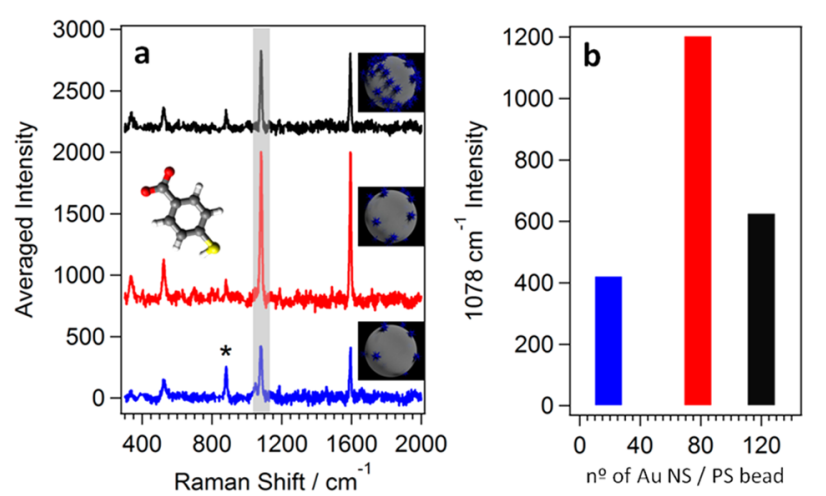

Figure 4. (a) SERS spectra of 4-MBA adsorbed on Au NSs-PS beads with different NS densities (blue $=$ low, red $=$ medium, black $=$ high). Spectra were background corrected and plotted after applying an offset for more convenient data presentation. The signal marked with $(*)$ is from the solvent of the 4-MBA stock (ethanol, $880 \mathrm{~cm}^{-1}$ ). (b) SERS intensity of 4-MBA breathing mode as a function of the average number of Au NSs per PS bead.

$$
\mathrm{EF}=\frac{I_{\mathrm{SERS}} N_{\text {bulk }}}{N_{\mathrm{ads}} I_{\text {Raman }}}
$$

where $I_{\text {SERS }}$ is the intensity of a vibrational mode in the SERS spectrum, $I_{\text {Raman }}$ the intensity of the same mode in the bulk Raman spectrum, $N_{\text {ads }}$ the number of molecules adsorbed onto the nanoparticle surface contributing to the SERS signal, and $N_{\text {bulk }}$ the number of molecules sampled in the bulk material. Ideally, the Raman spectrum of 4-MBA in aqueous solution would be used to determine the EF for Au NS-PS assemblies in solution, but no vibrational bands of 4-MBA could be observed under these conditions. Thus, the Raman spectrum of 4-MBA was measured from solid powder and used to normalize SERS spectra measured from a dried monolayer of PS beads with different $\mathrm{Au}$ NS coverage. The number of sampled 4-MBA molecules from the bulk was estimated by the method reported by Khan et al., ${ }^{53}$ whereas the number of sampled molecules from SERS was calculated from the Au NS surface available for each coverage degree within the laser illumination area, multiplied by the bonding density of $4-\mathrm{MBA}(\approx 0.5 \mathrm{nmol}$ $\left.\mathrm{cm}^{-2}\right),{ }^{54}$ assuming a complete monolayer of adsorbed molecules. Using the intensity of the $\nu(\mathrm{CC})_{\text {ring }}$ breathing (at $1102 \mathrm{~cm}^{-1}$ in bulk and at $1078 \mathrm{~cm}^{-1}$ for SERS samples) EFs can be estimated resulting in $7.3 \times 10^{7}$ (low), $7.5 \times 10^{7}$ (medium), and $1.8 \times 10^{7}$ (high) which is within the typical range between $10^{7}$ and $10^{8}$ for other gold nanoparticles. ${ }^{55} \mathrm{~A}$ more detailed presentation of the EF estimation is provided in the Supporting Information.

Detailed understanding of the SERS performance was achieved by means of theoretical modeling of the electric near field enhancement by the assembled nanostructures at different Au NS coverage. As the SIE-MoM formulation allows us to simulate the response of objects with arbitrarily complex morphologies, we used a model using a realistic Au NS distribution on the PS bead, as previously determined from TEM images (Figure 5a-c). Calculated (total) SERS intensity was averaged over p-polarized and s-polarized normal irradiation for particles containing different Au NS loadings (Figure 5e). For both low and medium Au NS coatings, the simulations indicate high electromagnetic field enhancement at the tips of every individual Au NS, with hardly any effects of plasmon coupling. Accordingly, the overall SERS intensity increases with the total number of Au NS tips, i.e., with the Au NS density. For high Au NS density, a different situation is however observed, where the intensity of electromagnetic field at tips within aggregated Au NS "islands" fades out, whereas hot spots remain at the boundaries only (Figure 5c, inset). The clusters thus behave as bigger particles with significantly reduced SERS activity at the resonant wavelength (Figure 5e, black curve). Indeed, this effect was not observed for simulations considering equally high Au NS loading but with a more uniform distribution over the PS sphere, i.e., no clustering (Figure 5d). The interparticle separation indeed 

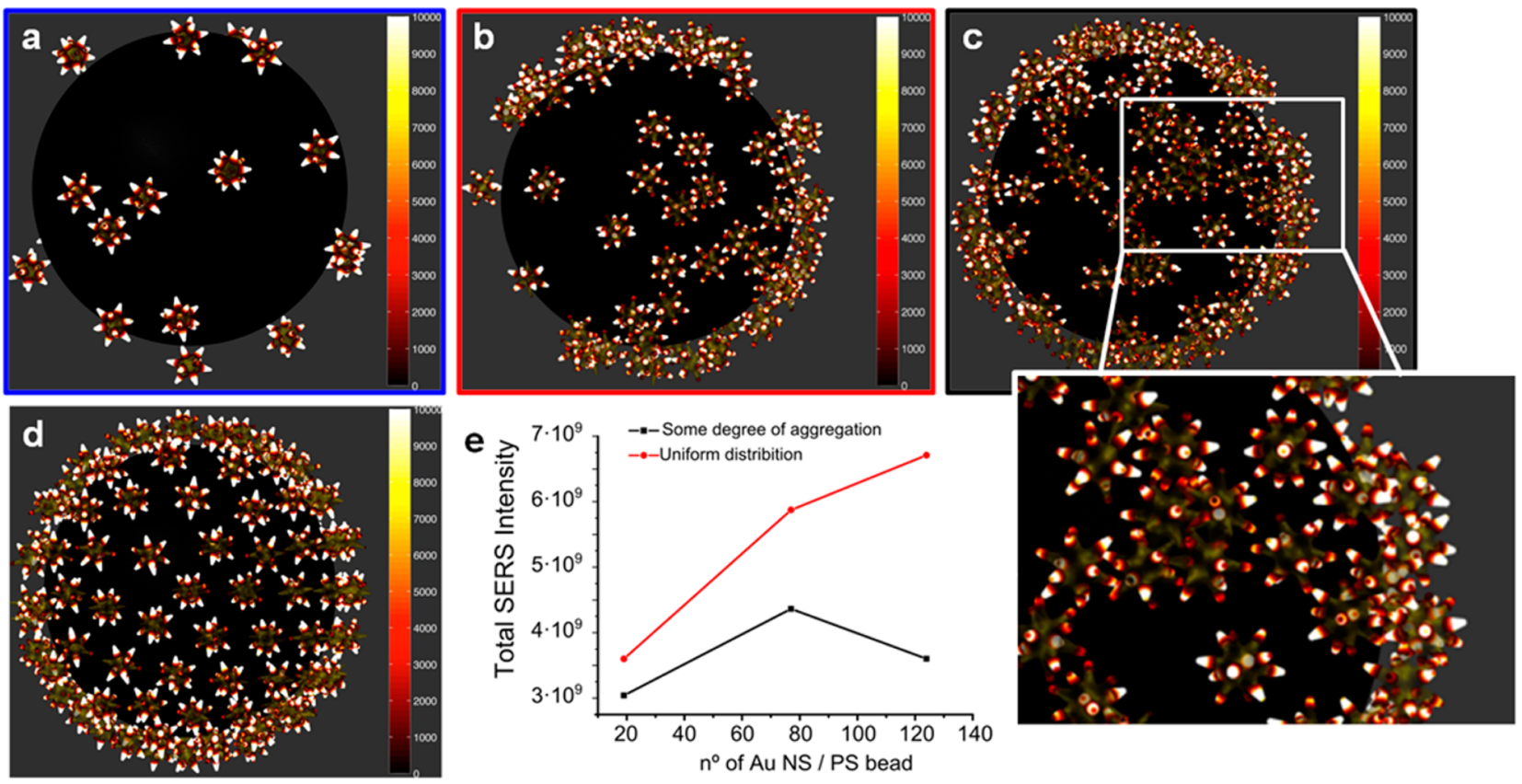

Figure 5. Calculated near field enhancement maps $(\lambda=785 \mathrm{~nm})$ of Au NS-PS particles. $(\mathrm{a}-\mathrm{c})$ Realistic modeling of Au NS-PS particles with low (a), medium (b), and high (c) Au NS loadings. (d) PS particles with high Au NS density and even distribution. (e) Total SERS intensity as a function $\mathrm{Au}$ NS density, for uniform Au NS distribution (red) and for partially aggregated Au NSs on PS, resembling the experimental observations (black).

avoids plasmon deactivation in this case, resulting in intense hot spots at all individual tips, so that the SERS intensity again increases with the number of $\mathrm{Au}$ NSs on the PS support, as observed for low and medium coverages (Figure 5e, red curve). The results of our simulations are therefore in good agreement with the experimental results and provide guidance toward engineering new Au NS-based substrates for SERS. Whereas isolated Au NSs preserve their "activated" sharp tips, we demonstrate that aggregation leads to strong plasmon coupling resulting in hot spot deactivation and therefore to lower SERS intensity. This may also be related to recent results showing that aggregation within endosomes hinders the efficiency of $\mathrm{Au}$ NSs toward photothermal therapy. ${ }^{56}$

As a result of experimental and theoretical optimization, we selected the most SERS-efficient configuration (4-MBA labeled $\mathrm{Au}$ NS-PS, medium-Au NS density) to demonstrate the applicability of these hybrid nanostructures for imaging of living cells. After centrifugation, the particles were successfully redispersed in CDMEM and incubated for $24 \mathrm{~h}$ to ensure stability in biological media, as indicated by no LSPR band alteration in the Vis-NIR spectra before and after incubation (Figure S5, SI). It is worth mentioning that neither tip reshaping nor $\mathrm{Au}$ NS detachment from the PS surface was observed. Successful in vitro SERS bioimaging requires that the nanotags are nontoxic and effectively uptaken by living cells. We evaluated the SERS efficiency of the hybrid particles as compared to free Au NSs. An equal amount of A549 cells was incubated either with 4-MBA-labeled Au NS-PS assemblies at a concentration of $7.5 \times 10^{-4}$ (\% PS in solids, $\left[\mathrm{Au}^{0}\right]=0.25 \mathrm{mM}$ ) or the equivalent concentration of free 4-MBA-labeled Au NSs. Figure 6 illustrates the corresponding 4-MBA SERS spectra and SERS maps obtained from both free Au NS and composite particles, upon internalization by A549 cells after $24 \mathrm{~h}$ incubation. The SERS fingerprint of 4-MBA was clearly identified, and no interference peaks originating from cells or
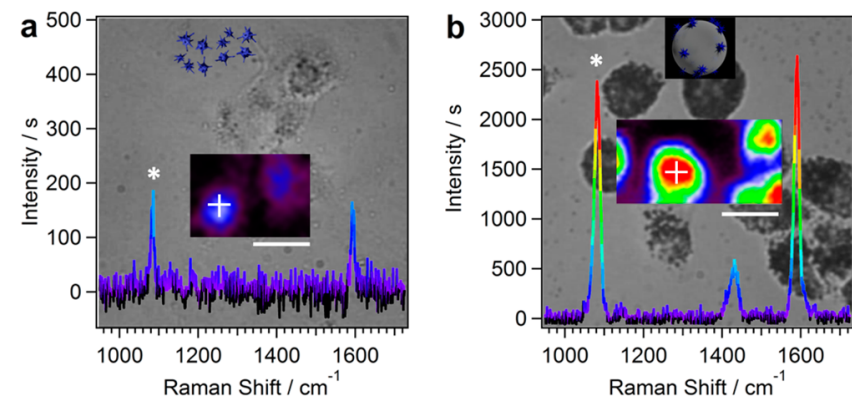

Figure 6. SERS maps and spectra from A549 cells incubated with 4MBA labeled Au NS (a) and Au NS-PS beads (b). The concentration of Au NS was constant in both cases and SERS spectra were baseline corrected. SERS maps were created by plotting the intensity of vibration at $1078 \mathrm{~cm}^{-1}$ (labeled with $*$ ) as a function of the $x, y$ position measured with step sizes of $2 \mu \mathrm{m}$ (a) and $1 \mu \mathrm{m}$ (b). The SERS spectra correspond to the points indicated with a white cross within each map. The intensity color scale for SERS spectra and 2D map in a was multiplied by 3 to create the SERS spectrum and map in b. White scale bars: $20 \mu \mathrm{m}$.

buffer solution under the present experimental conditions were observed. As compared to free Au NSs, the assembled nanotags yielded remarkably intense SERS signals, which may be related to enhanced uptake of NPs clustered on a delivery platform, as previously reported. ${ }^{53}$ In order to confirm the cellular uptake of both Au NS-PS and Au NS, TEM images were acquired after $12 \mathrm{~h}$ incubation with cells (Figure S6, SI). Both types of particles were successfully internalized by the cells, most likely by endocytosis, ${ }^{57}$ and importantly no sign was found of Au NSs that could have detached from the PS beads (Figure S6a, SI), indicating the high stability of the hybrid nanostructures. We additionally explored the general application regarding the choice of cell lines via SERS imaging of human MCF-7 breast cancer cells and murine J774 macrophages, with equally good 
results (Figure S7). This is an additional evidence that the assembled nanotags notably improve the possibilities of using $\mathrm{Au}$ NSs for SERS bioimaging.

Cell viability assays were also carried out, for which A549 cells were incubated with different concentrations of $\mathrm{Au} \mathrm{NSs}$, Au NS-PS, and PS particles for $66 \mathrm{~h}$ (Figure S8). Low levels of toxicity were noted, mainly at the highest concentration, which-given the long incubation time-may actually be due to a reduction in cell proliferation rather than a toxic effect of the nanoparticle system.

We finally present a proof of concept experiment where the hybrid nature of the particles was exploited to build multifunctional nanotags for multimodal bioimaging: fluorescence, SERS and dark field (DF) imaging. To this end, fluorescently labeled PS beads were used as colloidal support, so that complementary fluorescent imaging can be used to better understand relationship between cellular uptake, intracellular localization, and the recorded SERS signal. With a suitable strategy, a simple nanotag can be devised that enables us to independently monitor the nanoparticle, the supporting bead and the SERS label. Au NSs were thus assembled onto fluorescent PS beads, followed by functionalization with the 4MBA SERS label. The dye in the PS beads was selected so as to avoid interference of the fluorescence $(500-550 \mathrm{~nm}=$ green $)$ with the excitation wavelength for SERS $(785 \mathrm{~nm}=\mathrm{NIR})$. Additionally, the light scattering of nanoparticles depends on the material, size, and shape, ${ }^{59}$ which for $\mathrm{Au}$ NS allows detection under DF illumination conditions and application as a complementary cell imaging tool. The representative example shown in Figure 7 allows us to see for the same A549 cells the strong SERS signal originating from 4-MBA-encoded Au NS-PS composites (Figure 7a), the strong scattered light from both the metal NPs and the PS cores (Figure 7b), as well as the bright green emission originating from the fluorophore in the
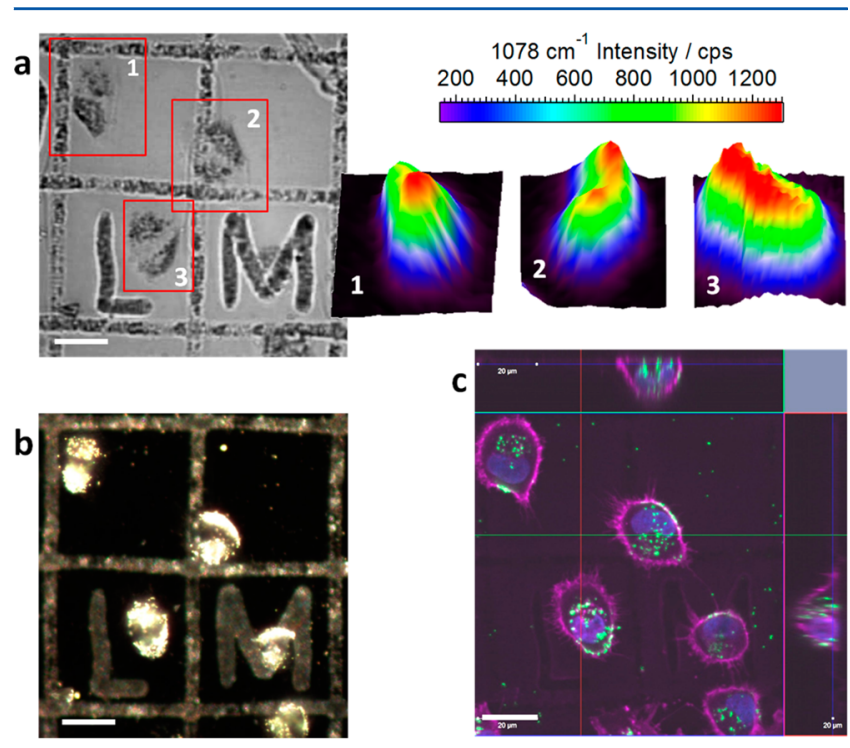

Figure 7. Multimodal imaging of living A549 cells using medium-Au NS-PS beads via correlated optical spectroscopy. (a) Bright-field micrograph and 3D-intensity maps of the $1078 \mathrm{~cm}^{-1}$ vibration of 4MBA in the SERS reporters, excited at $785 \mathrm{~nm}$, for three different cells. The spatial resolution of the SERS images was $2 \mu \mathrm{m}$ for 1 and $2,1 \mu \mathrm{m}$ for 3. (b) Dark field image of the same area. (c) Fluorescence mapping based on the emission $(500-550 \mathrm{~nm})$ from the fluorescently labeled PS beads. White scale bars: $20 \mu \mathrm{m}$. polymer beads (Figure 7c). The highest SERS intensities in Figure $7 \mathrm{a}$ clearly correlate with the brightest areas in the dark field image (Figure $7 \mathrm{~b}$ ), whereas the $z$-stack measurements in Figure $7 \mathrm{c}$ also support the intracellular presence of the nanocomposites. This example is a clear evidence of the high versatility of this composite particle system to separately track and image cellular objects using correlated microscopy/ spectroscopy tools. In a more realistic situation, the dual fluorescent-SERS tags could be labeled with several Raman reporter molecules and applied to multiplex biomarker detection.

\section{CONCLUSIONS}

A novel hybrid nanostructured system comprising $\mathrm{Au}$ NSs adsorbed on PS beads has been devised and fabricated, which shows great potential as multifunctional probe for bioimaging applications. Au NSs are highly efficient Raman-enhancing substrates which display LSPRs peaking in the biological transparency window. When assembled onto PS beads they present enhanced cell uptake, whereas the integration of various functionalities within the same composite particle allows us to image living cells using different analytical tools such as fluorescence, SERS and DF optical microscopy. Using 4-MBA as a Raman reporter molecule, the SERS efficiency of the hybrid composites containing different Au NS loadings was compared, obtaining for medium Au NS densities the highest SERS intensities. This intriguing finding was additionally supported by theoretical modeling, and we expect that the results presented here will open up new insights toward engineering of Au NS-based plasmonic nanostructures.

\section{ASSOCIATED CONTENT}

\section{S Supporting Information}

The Supporting Information is available free of charge on the ACS Publications website at DOI: 10.1021/acs.jpcc.6b02282.

More details on synthesis and assembly methods and characterization, sample preparation and TEM and SERS images of particles in cells, and cell viability assays of cells after incubation with particles (PDF)

\section{AUTHOR INFORMATION}

\section{Corresponding Author}

*E-mail: 1lizmarzan@cicbiomagune.es.

\section{Notes}

The authors declare no competing financial interest.

\section{ACKNOWLEDGMENTS}

Funding is acknowledged from the European Commission (Grant No. 310445-2 SAVVY), the European Research Council (ERC Advanced Grant No. 267867 Plasmaquo, and ERC Starting Grant No. 335078 Colouratom), and the Spanish MINECO (Project MAT2013-46101-R). We thank IKERLAT Polymers for the nonfluorescent PS beads and Prof. Juan Mareque, Prof. Soledad Penades, and Dr. Sergio Moya (CIC biomagune) for borrowing various cell lines. D.M.S., J.M.T, and F.O. acknowledge funding from the European Regional Development Fund (ERDF) and the Spanish MINECO (Projects MAT2014-58201-C2-1-R, MAT2014-58201-C2-2$\mathrm{R}$ ), from the ERDF and the Galician Regional Government under agreement for funding the Atlantic Research Center for Information and Communication Technologies (AtlantTIC), 
and from the ERDF and the Extremadura Regional Government (Junta de Extremadura) under Project IB13185.

\section{REFERENCES}

(1) Schlücker, S. Surface-Enhanced Raman Spectroscopy: Concepts and Chemical Applications. Angew. Chem., Int. Ed. 2014, 53, 47564795.

(2) Li, Z.-Y.; Xia, Y. Metal Nanoparticles with Gain toward SingleMolecule Detection by Surface-Enhanced Raman Scattering. Nano Lett. 2010, 10, 243-249.

(3) Stiles, P. L.; Dieringer, J. A.; Shah, N. C.; Van Duyne, R. P. Surface-Enhanced Raman Spectroscopy. Annu. Rev. Anal. Chem. 2008, $1,601-626$.

(4) McCreery, R. L. Frontmatter. In Raman Spectroscopy for Chemical Analysis; John Wiley \& Sons, Inc.: New York, USA, 2000.

(5) Wang, Y.; Yan, B.; Chen, L. SERS Tags: Novel Optical Nanoprobes for Bioanalysis. Chem. Rev. 2013, 113, 1391-1428.

(6) Kleinman, S. L.; Frontiera, R. R.; Henry, A.-I.; Dieringer, J. A.; Van Duyne, R. P. Creating, Characterizing, and Controlling Chemistry with SERS Hot Spots. Phys. Chem. Chem. Phys. 2013, 15, 21-36.

(7) Bryant, G. W.; García de Abajo, F. J.; Aizpurua, J. Mapping the Plasmon Resonances of Metallic Nanoantennas. Nano Lett. 2008, 8, 631-636.

(8) Nelayah, J.; Kociak, M.; Stéphan, O.; García de Abajo, F. J.; Tencé, M.; Henrard, L.; Taverna, D.; Pastoriza-Santos, I.; Liz-Marzán, L. M.; Colliex, C. Mapping Surface Plasmons on a Single Metallic Nanoparticle. Nat. Phys. 2007, 3, 348-353.

(9) Rodríguez-Lorenzo, L.; Álvarez-Puebla, R. A.; Pastoriza-Santos, I.; Mazzucco, S.; Stéphan, O.; Kociak, M.; Liz-Marzán, L. M.; García de Abajo, F. J. Zeptomol Detection Through Controlled Ultrasensitive Surface-Enhanced Raman Scattering. J. Am. Chem. Soc. 2009, 131, 4616-4618.

(10) Kumar, P. S.; Pastoriza-Santos, I.; Rodríguez-González, B.; García de Abajo, F. J.; Liz-Marzán, L. M. High-Yield Synthesis and Optical Response of Gold Nanostars. Nanotechnology 2008, 19, 15606.

(11) Guerrero-Martínez, A.; Barbosa, S.; Pastoriza-Santos, I.; LizMarzán, L. M. Nanostars Shine Bright for You. Curr. Opin. Colloid Interface Sci. 2011, 16, 118-127.

(12) Alvarez-Puebla, R.; Liz-Marzán, L. M.; García de Abajo, F. J. Light Concentration at the Nanometer Scale. J. Phys. Chem. Lett. 2010, 1, 2428-2434.

(13) Fabris, L.; Indrasekara, A. S. D. S.; Meyers, S.; Shubeita, S.; Feldman, L. C.; Gustafsson, T. Gold Nanostar Substrates for SERSBased Chemical Sensing in the Femtomolar Regime. Nanoscale 2014, 6, 8891-8899.

(14) Shiohara, A.; Langer, J.; Polavarapu, L.; Liz-Marzán, L. M. Solution Processed Polydimethylsiloxane/gold Nanostar Flexible Substrates for Plasmonic Sensing. Nanoscale 2014, 6, 9817-9823.

(15) Mir-Simon, B.; Morla-Folch, J.; Gisbert-Quilis, P.; Pazos-Perez, N.; Xie, H.; Bastús, N. G.; Puntes, V.; Alvarez-Puebla, R. A.; Guerrini, L. SERS Efficiencies of Micrometric Polystyrene Beads Coated with Gold and Silver Nanoparticles: The Effect of Nanoparticle Size. J. Opt. 2015, 17, 114012.

(16) Piskin, E.; Tuncel, A.; Denizli, A.; Ayhan, H. Monosize Microbeads Based on Polystyrene and Their Modified Forms for Some Selected Medical and Biological Applications. J. Biomater. Sci., Polym. Ed. 1994, 5, 451-471.

(17) Li, Y.; Pan, Y.; Zhu, L.; Wang, Z.; Su, D.; Xue, G. Facile and Controlled Fabrication of Functional Gold Nanoparticle-Coated Polystyrene Composite Particle. Macromol. Rapid Commun. 2011, 32, 1741-1747.

(18) Gong, J.; Zu, X.; Mu, W.; Deng, Y. In Situ Self-Assembly Synthesis of Gold Nanoparticle Arrays on Polystyrene Microspheres and Their Surface Plasmon Resonance. Colloid Polym. Sci. 2013, 291, 239-244.

(19) Zhu, W.; Wu, Y.; Yan, C.; Wang, C.; Zhang, M.; Wu, Z. Facile Synthesis of Mono-Dispersed Polystyrene (PS)/Ag Composite Microspheres via Modified Chemical Reduction. Materials 2013, 6, 5625-5638.
(20) Zhang, N.; Yu, X.; Hu, J.; Xue, F.; Ding, E. Synthesis of Silver Nanoparticle-Coated Poly(styrene-Co-Sulfonic Acid) Hybrid Materials and Their Application in Surface-Enhanced Raman Scattering (SERS) Tags. RSC Adv. 2013, 3, 13740-13747.

(21) Shi, W.; Sahoo, Y.; Swihart, M. T.; Prasad, P. N. Gold Nanoshells on Polystyrene Cores for Control of Surface Plasmon Resonance. Langmuir 2005, 21, 1610-1617.

(22) Guo, I. W.; Pekcevik, I. C.; Wang, M. C. P.; Pilapil, B. K.; Gates, B. D. Colloidal Core-shell Materials with "spiky" Surfaces Assembled from Gold Nanorods. Chem. Commun. 2014, 50, 8157-8160.

(23) Pilapil, B. K.; Wang, M. C. P.; Paul, M. T. Y.; Nazemi, A.; Gates, B. D. Self-Assembly of Nanoparticles onto the Surfaces of Polystyrene Spheres with a Tunable Composition and Loading. Nanotechnology 2015, 26, 55601.

(24) Pallaoro, A.; Braun, G. B.; Reich, N. O.; Moskovits, M. Mapping Local $\mathrm{pH}$ in Live Cells Using Encapsulated Fluorescent SERS Nanotags. Small 2010, 6, 618-622.

(25) Niu, X.; Chen, H.; Wang, Y.; Wang, W.; Sun, X.; Chen, L. Upconversion Fluorescence-SERS Dual-Mode Tags for Cellular and in Vivo Imaging. ACS Appl. Mater. Interfaces 2014, 6, 5152-5160.

(26) Le Ru, E. C.; Etchegoin, P. G. Raman Spectroscopy and Related Optical Techniques. In Principles of Surface-Enhanced Raman Spectroscopy; Elsevier: Amsterdam, 2009; pp 29-120.

(27) Schlücker, S. SERS Microscopy: Nanoparticle Probes and Biomedical Applications. ChemPhysChem 2009, 10, 1344-1354.

(28) Lee, S.; Chon, H.; Yoon, S.-Y.; Lee, E. K.; Chang, S.-I.; Lim, D. W.; Choo, J. Fabrication of SERS-Fluorescence Dual Modal Nanoprobes and Application to Multiplex Cancer Cell Imaging. Nanoscale 2012, 4, 124-129.

(29) Zhang, Y.; Qian, J.; Wang, D.; Wang, Y.; He, S. Multifunctional Gold Nanorods with Ultrahigh Stability and Tunability for In Vivo Fluorescence Imaging, SERS Detection, and Photodynamic Therapy. Angew. Chem., Int. Ed. 2013, 52, 1148-1151.

(30) Yu, K. N.; Lee, S.-M.; Han, J. Y.; Park, H.; Woo, M.-A.; Noh, M. S.; Hwang, S.-K.; Kwon, J.-T.; Jin, H.; Kim, Y.-K.; et al. Multiplex Targeting, Tracking, and Imaging of Apoptosis by Fluorescent Surface Enhanced Raman Spectroscopic Dots. Bioconjugate Chem. 2007, 18, $1155-1162$.

(31) Qian, J.; Jiang, L.; Cai, F.; Wang, D.; He, S. FluorescenceSurface Enhanced Raman Scattering Co-Functionalized Gold Nanorods as near-Infrared Probes for Purely Optical in Vivo Imaging. Biomaterials 2011, 32, 1601-1610.

(32) Wang, Z.; Zong, S.; Yang, J.; Li, J.; Cui, Y. Dual-Mode Probe Based on Mesoporous Silica Coated Gold Nanorods for Targeting Cancer Cells. Biosens. Bioelectron. 2011, 26, 2883-2889.

(33) Chen, G.; Teng, Z.; Su, X.; Liu, Y.; Lu, G. Unique Biological Degradation Behavior of Stöber Mesoporous Silica Nanoparticles from Their Interiors to Their Exteriors. J. Biomed. Nanotechnol. 2015, 11, $722-729$.

(34) Izak-Nau, E.; Voetz, M.; Eiden, S.; Duschl, A.; Puntes, V. F. Altered Characteristics of Silica Nanoparticles in Bovine and Human Serum: The Importance of Nanomaterial Characterization prior to Its Toxicological Evaluation. Part. Fibre Toxicol. 2013, 10, 56.

(35) Palenstijn, W. J.; Batenburg, K. J.; Sijbers, J. Performance Improvements for Iterative Electron Tomography Reconstruction Using Graphics Processing Units (GPUs). J. Struct. Biol. 2011, 176, 250-253.

(36) Yuan, H.; Khoury, C. G.; Hwang, H.; Wilson, C. M.; Grant, G. A.; Vo-Dinh, T. Gold Nanostars: Surfactant-Free Synthesis, 3D Modelling, and Two-Photon Photoluminescence Imaging. Nanotechnology 2012, 23, 75102.

(37) Solís, D. M.; Taboada, J. M.; Obelleiro, F.; Liz-Marzán, L. M.; García de Abajo, F. J. Toward Ultimate Nanoplasmonics Modeling. ACS Nano 2014, 8, 7559-7570.

(38) Solís, D. M.; Taboada, J. M.; Basteiro, F. O. Surface Integral Equation-Method of Moments With Multiregion Basis Functions Applied to Plasmonics. IEEE Trans. Antennas Propag. 2015, 63, 21412152. 
(39) Taboada, J. M.; Araujo, M. G.; Basteiro, F. O.; Rodriguez, J. L.; Landesa, L. MLFMA-FFT Parallel Algorithm for the Solution of Extremely Large Problems in Electromagnetics. Proc. IEEE 2013, 101, 350-363.

(40) Solís, D. M.; Araújo, M. G.; Landesa, L.; García, S.; Taboada, J. M.; Obelleiro, F. MLFMA-MoM for Solving the Scattering of Densely Packed Plasmonic Nanoparticle Assemblies. IEEE Photonics J. 2015, 7, $1-9$.

(41) Bastús, N. G.; Merkoçi, F.; Piella, J.; Puntes, V. Synthesis of Highly Monodisperse Citrate-Stabilized Silver Nanoparticles of up to 200 nm: Kinetic Control and Catalytic Properties. Chem. Mater. 2014, 26, 2836-2846.

(42) Barbosa, S.; Agrawal, A.; Rodríguez-Lorenzo, L.; PastorizaSantos, I.; Alvarez-Puebla, R. A.; Kornowski, A.; Weller, H.; LizMarzán, L. M. Tuning Size and Sensing Properties in Colloidal Gold Nanostars. Langmuir 2010, 26, 14943-14950.

(43) McLellan, J. M.; Siekkinen, A.; Chen, J.; Xia, Y. Comparison of the Surface-Enhanced Raman Scattering on Sharp and Truncated Silver Nanocubes. Chem. Phys. Lett. 2006, 427, 122-126.

(44) Shiohara, A.; Wang, Y.; Liz-Marzán, L. M. Recent Approaches toward Creation of Hot Spots for SERS Detection. J. Photochem. Photobiol., C 2014, 21, 2-25.

(45) Vega, M. M.; Bonifacio, A.; Lughi, V.; Marsi, S.; Carrato, S.; Sergo, V. Long-Term Stability of Surfactant-Free Gold Nanostars. J. Nanopart. Res. 2014, 16, 1-6.

(46) Serrano-Montes, A. B.; Jimenez de Aberasturi, D.; Langer, J.; Giner-Casares, J. J.; Scarabelli, L.; Herrero, A.; Liz-Marzán, L. M. A General Method for Solvent Exchange of Plasmonic Nanoparticles and Self-Assembly into SERS-Active Monolayers. Langmuir 2015, 31, 9205-9213.

(47) Yuan, H.; Fales, A. M.; Khoury, C. G.; Liu, J.; Vo-Dinh, T. Spectral Characterization and Intracellular Detection of SurfaceEnhanced Raman Scattering (SERS)-Encoded Plasmonic Gold Nanostars. J. Raman Spectrosc. 2013, 44, 234-239.

(48) Jana, N. R.; Gearheart, L.; Murphy, C. J. Seeding Growth for Size Control of 5-40 nm Diameter Gold Nanoparticles. Langmuir 2001, 17, 6782-6786.

(49) Michota, A.; Bukowska, J. Surface-Enhanced Raman Scattering (SERS) of 4-Mercaptobenzoic Acid on Silver and Gold Substrates. J. Raman Spectrosc. 2003, 34, 21-25.

(50) Gole, A.; Orendorff, C. J.; Murphy, C. J. Immobilization of Gold Nanorods onto Acid-Terminated Self-Assembled Monolayers via Electrostatic Interactions. Langmuir 2004, 20, 7117-7122.

(51) Aldeanueva-Potel, P.; Carbó-Argibay, E.; Pazos-Pérez, N.; Barbosa, S.; Pastoriza-Santos, I.; Alvarez-Puebla, R. A.; Liz-Marzán, L. M. Spiked Gold Beads as Substrates for Single-Particle SERS. ChemPhysChem 2012, 13, 2561-2565.

(52) Toderas, F.; Baia, M.; Baia, L.; Astilean, S. Controlling Gold Nanoparticle Assemblies for Efficient Surface-Enhanced Raman Scattering and Localized Surface Plasmon Resonance Sensors. Nanotechnology 2007, 18, 255702.

(53) Khan, M. A.; Hogan, T. P.; Shanker, B. Surface-Enhanced Raman Scattering from Gold-Coated Germanium Oxide Nanowires. J. Raman Spectrosc. 2008, 39, 893-900.

(54) Taylor, C. E.; Pemberton, J. E.; Goodman, G. G.; Schoenfisch, M. H. Surface Enhancement Factors for Ag and Au Surfaces Relative to Pt Surfaces for Monolayers of Thiophenol. Appl. Spectrosc. 1999, 53, $1212-1221$

(55) Orendorff, C. J.; Gole, A.; Sau, T. K.; Murphy, C. J. SurfaceEnhanced Raman Spectroscopy of Self-Assembled Monolayers: Sandwich Architecture and Nanoparticle Shape Dependence. Anal. Chem. 2005, 77, 3261-3266.

(56) Espinosa, A.; Silva, A. K. A.; Sánchez-Iglesias, A.; Grzelczak, M.; Péchoux, C.; Desboeufs, K.; Liz-Marzán, L. M.; Wilhelm, C. Cancer Cell Internalisation of Gold Nanostars Impacts Their Photothermal Efficiency in Vitro and in Vivo: Towards a Plasmonic Thermal Fingerprint in Tumoral Environment. Adv. Healthcare Mater. 2016, 5, 1040-1048.
(57) Nativo, P.; Prior, I. A.; Brust, M. Uptake and Intracellular Fate of Surface-Modified Gold Nanoparticles. ACS Nano 2008, 2, 16391644.

(58) Jia, H.-Z.; Chen, W.-H.; Wang, X.; Lei, Q.; Yin, W.-N.; Wang, Y.; Zhuo, R.-X.; Feng, J.; Zhang, X.-Z. Virus-Surface-Mimicking Surface Clustering of AuNPs onto DNA-Entrapped Polymeric Nanoparticle for Enhanced Cellular Internalization and NanoclusterInduced NIR Photothermal Therapy. Adv. Sci. 2015, 2, 1500108.

(59) Jain, P. K.; Lee, K. S.; El-Sayed, I. H.; El-Sayed, M. A. Calculated Absorption and Scattering Properties of Gold Nanoparticles of Different Size, Shape, and Composition: Applications in Biological Imaging and Biomedicine. J. Phys. Chem. B 2006, 110, 7238-7248. 